\title{
El aprendizaje-servicio: una herramienta en la formación del profesorado en clave intercultural. Construyendo puentes entre Ecuador y Euskadi
}

\author{
Imanol Santamaría-Goicuria \\ Irune Corres-Medrano \\ Joseba Iñaki Arregi-Orue \\ Universidad del País Vasco-Euskal Herriko Unibertsitatea, España
}

\section{Resumen}

Teniendo como objetivo responder a la pregunta: ¿cuál es la contribución que la implementación de la metodología aprendizaje-servicio tiene en la adquisición de competencias interculturales de nuestro alumnado de magisterio y del futuro profesorado?, esta experiencia, se desarrolló durante el curso 2016-2017 y participaron un total de 54 alumnos de la Facultad de Educación y Deporte de la Universidad del País Vasco de Vitoria-Gasteiz. Como resultado, se evidencia que los futuros docentes demandaban experimentar un modelo de formación más activa y verdaderamente comprometida con la sociedad y con ellos mismos en la que además de desarrollar sus competencias interculturales, se pudieran desarrollar también como personas justas, críticas comprometidas con la comunidad, indistintamente del contexto de su localización. Se concluye que la utilización de este tipo de metodologías activas como el aprendizaje-servicio poseen un gran valor pedagógico, social, democrático y formativo del alumnado porque incide en sus aspectos personales y profesionales, favoreciendo el desarrollo de su competencia intercultural y aumentando su nivel de compromiso comunitario, valores estos fundamentales.

\section{Palabras clave}

Aprendizaje-servicio, futuros educadores, competencias interculturales.

Fecha de recepción: 1/III/2021

Fecha de aceptación: 26/III/2021

Santamaría-Goicuria, I.; Corres-Medrano, I. y Arregi-Orue, J. (2021). El aprendizaje-servicio: una herramienta en la formación del profesorado en clave intercultural. Construyendo puentes entre Ecuador y Euskadi. RIDAS, Revista Iberoamericana de Aprendizaje Servicio, 11, 139-152.

DOI10.1344/RIDAS2021.11.10 


\title{
Service-learning: a tool for intercultural teacher training. Building bridges between Ecuador and the Basque Country
}

\begin{abstract}
With the aim of analysing the benefits of the service-learning methodology in the acquisition of intercultural competences of teacher training students and pre-service teachers, an experience was carried out during the 2016-2017 academic year. A total of 54 students from the Faculty of Education and Sport of the University of the Basque Country in Vitoria-Gasteiz took part. The results showed that pre-service teachers demanded to experience a more active training model that was truly committed to society and to themselves and through which they could not only develop their intercultural competences, but they could also grow as fair, critical people, committed to the community, regardless of the context of the learning experience. The paper concludes that the use of active methodologies such as service-learning has a significant pedagogical, social, democratic and formative value for students as it impacts on their personal and professional development, fostering the development of their intercultural competence and increasing their level of community engagement, which are all essential values.
\end{abstract}

\section{Keywords}

Service-learning, future educators, intercultural competences. y Euskadi. RIDAS, Revista Iberoamericana de Aprendizaje Servicio, 11, 139-152.

DOI10.1344/RIDAS2021.11.10 


\section{Introducción}

En la actualidad el mundo se enfrenta a grandes desafíos que cuestionan radicalmente los fundamentos teóricoprácticos y onto-epistemológicos sobre los que se han desarrollado numerosas generaciones precedentes. Esta incertidumbre que genera la transición de la 'antigua normalidad' a la 'nueva normalidad' supone la construcción de una sociedad en donde el saber que se produce, distribuye y analiza es resbaladizo y las interpretaciones de esta complejidad dinámica caducan rápidamente.

Este escenario surgido de las transformaciones socio-culturales de las últimas décadas invitan a desarrollar una renovación de las prácticas pedagógicas utilizadas en la educación en general (Giroux, 2019) y más concretamente en los procesos de enseñanza-aprendizaje y la formación del profesorado en la universidad. Se necesita una universidad global con elementos educativos para una formación en clave intercultural, cuya oferta formativa considere diversos campos profesionales, que facilite acciones de servicio a la comunidad para erradicar situaciones de injusticia y de desigualdad social y desarrollar así, una verdadera convivencia intercultural (Calatayud, 2006; Francisco y Moliner, 2010; Palomero, 2006). En definitiva, se demanda una universidad como espacio y actor transformador de la sociedad (Francisco y Moliner, 2010).

Tomando como punto de partida las conclusiones obtenidas a través del diálogo vasco-ecuatoriano desarrollado en las jornadas universitarias realizadas en 2016 sobre la realidad de la Educación Intercultural Bilingüe en Ecuador (Arregi y Orbea, 2016), este trabajo se centra en el aprendizajeservicio.

El aprendizaje-servicio, como herramienta transformadora, posibilita el desarrollo de sociedades pues proporcionan la adquisición de competencias a través de la transmisión de conocimientos y dan lugar a las relacionadas con aspectos emocionales primordiales en la construcción social y el desarrollo humano (Delors, 1996).

\subsection{Competencia intercultural}

El mundo globalizado actual interconecta a todas las culturas del planeta de una forma radical, íntima y compleja de la que es imposible escapar (Arregi 2011). En esta segunda década del siglo XXI, se generan múltiples experiencias de encuentrodesencuentro cultural, pero a pesar de las dificultades que esto entraña, el capitalismo neoliberal puede ser combatido pudiéndose derrotar algunos de sus peores rasgos (Chomsky et al., 2015). La competencia intercultural se convierte en fundamental a la hora de plantear una educación para un mundo globalizado.

La competencia intercultural es la capacidad de desarrollar conocimientos, aptitudes y actitudes específicas que conduzcan a un comportamiento y una comunicación visibles que sean a la vez eficaces y apropiados en las interacciones interculturales, siendo el

Santamaría-Goicuria, I.; Corres-Medrano, I. y Arregi-Orue, J. (2021). El aprendizaje-servicio: una herramienta en la formación del profesorado en clave intercultural. Construyendo puentes entre Ecuador y Euskadi. RIDAS, Revista Iberoamericana de Aprendizaje Servicio, 11, 139-152. 


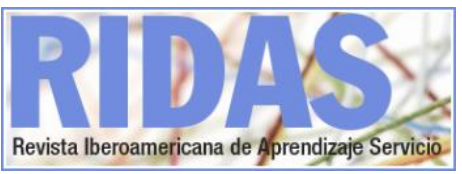

desarrollo de esta competencia un proceso complejo y duradero (Deardoff, 2006). McKinnon (2012) identifica elementos relacionados con tres áreas clave (conocimientos, aptitudes y actitudes) que son elementales para nuestra propuesta de aprendizajeservicio que se presenta a continuación en la tabla 1.

Tabla 1. Propuesta de aprendizaje y servicio basada en McKinnon (2012)

\begin{tabular}{|c|c|c|}
\hline Áreas Clave & $\begin{array}{c}\text { Elementos } \\
\text { fundamentales }\end{array}$ & Definición \\
\hline & $\begin{array}{l}\text { el conocimiento } \\
\text { específico de la } \\
\text { cultura }\end{array}$ & $\begin{array}{l}\text { la capacidad para } \\
\text { analizar y explicar } \\
\text { información básica } \\
\text { sobre otras culturas } \\
\text { (historia, valores, } \\
\text { política, economía, } \\
\text { estilos de } \\
\text { comunicación, } \\
\text { creencias y } \\
\text { prácticas) }\end{array}$ \\
\hline $\begin{array}{l}\text { La importancia } \\
\text { del } \\
\text { conocimiento } \\
\text { (autoconcien- } \\
\text { cia cultural) } \\
\text { en el proceso } \\
\text { de } \\
\text { construcción } \\
\text { de identidad }\end{array}$ & $\begin{array}{l}\text { la conciencia } \\
\text { sociolingüística }\end{array}$ & $\begin{array}{l}\text { conocimientos } \\
\text { básicos del idioma } \\
\text { local, articular las } \\
\text { diferencias en la } \\
\text { comunicación } \\
\text { verbal/no verbal y } \\
\text { ajustar el habla para } \\
\text { acomodar a los } \\
\text { nacionales de otras } \\
\text { culturas y la } \\
\text { comprensión de las } \\
\text { cuestiones y } \\
\text { tendencias } \\
\text { mundiales, es decir, } \\
\text { la capacidad para } \\
\text { explicar el significad } \\
\text { y las implicaciones } \\
\text { de la globalización y } \\
\text { la relaciones de las } \\
\text { cuestiones locales } \\
\text { con las fuerzas } \\
\text { mundiales }\end{array}$ \\
\hline
\end{tabular}

\begin{tabular}{|c|c|c|}
\hline \multirow{3}{*}{$\begin{array}{l}\text { La importancia } \\
\text { de las } \\
\text { aptitudes }\end{array}$} & $\begin{array}{l}\text { las } \\
\text { competencias } \\
\text { para escuchar, } \\
\text { observar, } \\
\text { evaluar }\end{array}$ & $\begin{array}{l}\text { que son las que } \\
\text { requieren de } \\
\text { paciencia y } \\
\text { perseverancia para } \\
\text { identificar y reducir } \\
\text { al mínimo el } \\
\text { etnocentrismo, } \\
\text { buscar pistas y } \\
\text { significados } \\
\text { culturales }\end{array}$ \\
\hline & $\begin{array}{l}\text { las } \\
\text { competencias } \\
\text { para analizar, } \\
\text { interpretar y } \\
\text { relacionar }\end{array}$ & $\begin{array}{l}\text { éstas estarían } \\
\text { basadas en la } \\
\text { búsqueda de } \\
\text { vínculos, causalidad } \\
\text { y relaciones } \\
\text { mediante técnicas de } \\
\text { análisis comparativo }\end{array}$ \\
\hline & $\begin{array}{l}\text { el pensamiento } \\
\text { crítico }\end{array}$ & $\begin{array}{l}\text { el cual conlleva la } \\
\text { capacidad de ver e } \\
\text { interpretar el mundo } \\
\text { desde el punto de } \\
\text { vista de otras } \\
\text { culturas e identificar } \\
\text { la propia }\end{array}$ \\
\hline \multirow{3}{*}{$\begin{array}{l}\text { La importancia } \\
\text { de las } \\
\text { actitudes }\end{array}$} & $\begin{array}{l}\text { el respeto hacia } \\
\text { los atributos de } \\
\text { otras culturas }\end{array}$ & $\begin{array}{l}\text { conlleva valorar la } \\
\text { diversidad cultural y } \\
\text { pensar } \\
\text { comparativamente y } \\
\text { sin prejuicios sobre } \\
\text { las diferencias } \\
\text { culturales; la } \\
\text { apertura, es decir, la } \\
\text { suspensión de la } \\
\text { crítica a otras } \\
\text { culturas }\end{array}$ \\
\hline & la curiosidad & $\begin{array}{l}\text { la cual promueve la } \\
\text { búsqueda de } \\
\text { interacciones } \\
\text { interculturales, } \\
\text { viendo la diferencia } \\
\text { como una } \\
\text { oportunidad de } \\
\text { aprendizaje y siendo } \\
\text { consciente de la } \\
\text { propia ignorancia }\end{array}$ \\
\hline & $\begin{array}{l}\text { la actitud de } \\
\text { descubrimiento }\end{array}$ & $\begin{array}{l}\text { supone la capacidad } \\
\text { de tolerancia de la } \\
\text { ambigüedad y } \\
\text { considerándola como } \\
\text { una experiencia } \\
\text { positiva, y por otro } \\
\text { lado, la voluntad de } \\
\text { ir más allá de la } \\
\text { propia zona de } \\
\text { confort }\end{array}$ \\
\hline
\end{tabular}

Fuente: elaboración propia

Santamaría-Goicuria, I.; Corres-Medrano, I. y Arregi-Orue, J. (2021). El aprendizaje-servicio: una herramienta en la formación del profesorado en clave intercultural. Construyendo puentes entre Ecuador y Euskadi. RIDAS, Revista Iberoamericana de Aprendizaje Servicio, 11, 139-152.

DOI10.1344/RIDAS2021.11.10 


\subsection{Creando puentes entre Ecuador y Euskadi}

El diálogo intercultural requiere de la participación de múltiples actores (Essomba, 2015) y de aproximaciones imaginativas que consigan generar confianza, empatía y complicidad entre todos los participantes en el proceso de convivencia intercultural.

La presencia indígena en nuestras aulas, predominantemente de indígenas otavalos de Ecuador, necesita de aproximaciones teóricas y métodos de acogida e integración innovadores para afrontar satisfactoriamente la enorme diversidad que hay que gestionar por ser España signataria del Convenio 169 de la Organización Internacional del Trabajo (Arregi y Orbea, 2016).

Inspiradas por estas ideas, en 2016 se plantearon unas jornadas para abordar las realidades y retos dentro de las cuales se realizó un seminario de una semana de duración sobre la realidad de la Educación Intercultural Bilingüe en Ecuador.

Durante la celebración del seminario, educadores indígenas de la Amazonia y Sierra ecuatorianas, presentaron la realidad de la Educación Intercultural Indígena en Ecuador, centrando su presentación en la centralidad del Sumak Kawsay (SMK) con el fin de generar un conflicto cognitivo, capaz de reconsiderar narrativas y despertar su espíritu crítico, a través de un aprendizaje dialógico (Arregi et al., 2017). En el desarrollo del seminario los estudiantes destacaron la crítica de la competición, el individualismo, la insolidaridad, la productividad y la falta de preocupación sobre los efectos que quiebran comunidades y ecosistemas en nombre de un progreso que les ha resultado históricamente esquivo a los pueblos indígenas.

En la base de este modelo alternativo (SMK) se encuentran el 'ayllu' (comunidad vinculada a ecosistemas y territorios concretos, base de la identidad individual y comunitaria) y el principio de reciprocidad y búsqueda del bien común (Arregi et al., 2018). En los debates con los docentes indígenas en torno a las claves de la Educación Intercultural se destacó que aplicar tales principios a nuestro contexto supone una apuesta por modelos de aprendizaje y acción social vinculados a la comunidad, mediante el aprendizajeservicio.

1.3 La universidad y su responsabilidad en el servicio a la comunidad: el aprendizaje-servicio para las competencias interculturales

La universidad, como institución educativa implicada con el desarrollo social, se establece sobre la responsabilidad social (Martínez, 2008). La responsabilidad social es entendida como la capacidad que tiene la universidad de difundir y poner en práctica un conjunto de principios y valores generales o específicos, por medio de diferentes procesos, en respuesta a las necesidades de la comunidad, implementando estrategias capaces de mejorar el devenir de las comunidades donde se encuentran presentes (Alonso-Sáez et al., 2015; De Ferari, 2006). Por tanto, la formación universitaria, no solo contempla contenidos teóricos y metodológicos,

Santamaría-Goicuria, I.; Corres-Medrano, I. y Arregi-Orue, J. (2021). El aprendizaje-servicio: una herramienta en la formación del profesorado en clave intercultural. Construyendo puentes entre Ecuador y Euskadi. RIDAS, Revista Iberoamericana de Aprendizaje Servicio, 11, 139-152. 
sino que también introduce contenidos de naturaleza real fundamentados en la capacidad de crítica, de reflexión y de participación activa obtenidos del contacto con un contexto real (Essomba, 2015; Puig, 2009).

Algunos de estos cambios vienen promovidos por la implementación del aprendizaje-servicio, ya que se trata de una metodología dinámica, en constante construcción. Es una metodología activa que promueve el desarrollo de los aprendizajes en diversos contextos, por lo que complementa la formación ofrecida en las aulas, aportando una experiencia vivencial y la posibilidad de desarrollar las áreas de competencia intercultural necesarias (Deardoff, 2006).

Existen numerosos trabajos que respaldan su uso (Aramburuzabala y García, 2012; Lucas y Martínez-Odria, 2012; Rubio y Escofet, 2018). Todos estos trabajos subrayan el gran impacto que genera el uso de esta metodología, principalmente en: el rendimiento académico del alumnado; la adquisición de las competencias curriculares; el desarrollo de las habilidades socioemocionales y desarrollo de la responsabilidad social (Martínez et al., 2013).

En consecuencia, se considera que el aprendizaje-servicio es un instrumento imprescindible en la adquisición de competencias interculturales durante la formación del futuro profesorado al ser una metodología basada en la experiencia que integra el aprendizaje y el servicio a la comunidad en una única acción (Sotelino et al., 2016).
La pregunta en la que se centra esta experiencia es la siguiente: ¿cuál es la contribución que la implementación de la metodología aprendizaje-servicio tiene en la adquisición de competencias interculturales de nuestro alumnado de magisterio y del futuro profesorado?

\section{Objetivo y contexto de la experiencia}

El objetivo de esta experiencia fue analizar la repercusión que la implementación de metodologías activas como el aprendizaje-servicio tuvo en la aportación pedagógica a la formación del profesorado en general y más concretamente a la adquisición de competencias interculturales.

Las personas participantes en este estudio pertenecían a diferentes comunidades y contextos educativos. Por un lado, participaron el alumnado y el profesorado de $4^{\circ}$ curso del Grado de Magisterio en Educación Infantil y Primaria en la mención de Educación Intercultural. Por otro lado, también colaboró el alumnado y profesorado de distintas instituciones escolares de Educación Primaria y de Educación Secundaria Obligatoria de la localidad de Vitoria-Gasteiz. Y en último lugar, participaron personas colaboradoras en asociaciones de colectivos migrantes, trabajadores y trabajadoras sociales del ayuntamiento de Vitoria-Gasteiz y las personas residentes en los diferentes barrios en los que se ha llevado a cabo la actividad en la que se basa este trabajo de investigación.

Para recopilar los datos se utilizaron diferentes herramientas como el y Euskadi. RIDAS, Revista Iberoamericana de Aprendizaje Servicio, 11, 139-152. 
portafolios (google sites) y las entrevistas semiestructuradas. En el transcurso del proceso, el alumnado elaboró un portafolio donde pudo recoger, a modo de cuaderno de bitácora, las diferentes evidencias (testimonios, imágenes, reflexiones...) obtenidas en las fases de identificación de necesidades e implementación.

\section{Desarrollo y análisis de resultados}

Los datos obtenidos, de naturaleza cualitativa, surgieron a partir del análisis de las fuentes de información utilizadas: entrevistas semiestructuradas y narrativas autobiográficas recogidas en los diferentes portafolios sobre el desarrollo de las diferentes fases que conformaron el proceso de aprendizajeservicio llevado a cabo.

La investigación se dividió en 4 fases: contextualización del aprendizajeservicio; visibilización de necesidades y desarrollo de las propuestas; explicación del proyecto implementado y evaluación del proceso.

\subsection{Fase I: contextualización del aprendizaje-servicio}

En la primera fase, en un inicio, se profundizó en los conceptos fundamentales del aprendizaje-servicio, precisando sus características particulares respecto a su metodología (Puig et al., 2007; 2008; RMC Research Corporation, 2006). Por lo que en esta fase se realizó una primera toma de contacto con el trabajo de campo y la metodología a desarrollar.
Para ello el profesorado presentó los principios básicos del programa en cuestión: definir el lugar donde se iba a desarrollar la actividad; temporalizar la actividad (duración total; creación de los horarios, etc.) y formar los grupos de trabajo determinar las zonas de trabajo de cada uno de ellos.

Finalmente, se les explicó que previamente a la actividad, cada grupo debía analizar de manera exhaustiva el contexto, poniendo la atención sobre todo en la diversidad étnico-cultural de la comunidad en cuestión, para a continuación poder realizar un análisis descriptivo sobre las relaciones entre las personas miembro de esas comunidades y poder así detectar y determinar si existen necesidades específicas por cubrir en aras de mejorar la calidad entre relaciones.

\subsection{Fase II: visibilización de} necesidades y desarrollo de las propuestas

La segunda fase se llevó a cabo en dos periodos: el de la identificación de necesidades y el desarrollo de intervenciones. Tras la identificación, el objetivo era ofertar un servicioproyecto que respondiera a dichas necesidades. Para la identificación de necesidades se utilizó una muestra de conveniencia (Polkinghorne, 2005) a través de entrevistas semiestructuradas.

De igual modo, se realizaron entrevistas a diferentes personas vecinas siguiendo la misma técnica de muestreo. De esta manera, el alumnado obtuvo relatos de diversas personas vecinas de este barrio en y Euskadi. RIDAS, Revista Iberoamericana de Aprendizaje Servicio, 11, 139-152. 
torno a la convivencia entre las diferentes culturas. En general, las personas residentes en este barrio tenían conciencia sobre su diversidad étnico-cultural y hablaban de respeto, señalando que convivían diferentes comercios específicos para satisfacer las necesidades de cada colectivo: carnicerías Halal, locutorios, etc. A través de las entrevistas se recogió información relacionada con diferentes culturas: pakistaníes, marroquíes, gitanos, senegaleses, vascos, etc.

Sin embargo, la mayoría de las personas entrevistadas consideró que, a pesar de la amplísima diversidad cultural, no había interculturalidad, sino que convivían ghettos diferentes. Incluso se mencionaron sucesos de índole xenófobo. Por lo tanto, una demanda recurrente fue la implementación de nuevos recursos que promovieran la convivencia entre las diferentes culturas y así enriquecer las relaciones entre las personas.

\subsection{Fase III: desarrollo del proyecto implementado}

En esta fase se explicó el proceso de implementación de la intervención, basada en las necesidades identificadas en la fase anterior (fase II). La principal deficiencia detectada fue la ausencia de relaciones entre las personas de diferentes orígenes étnico-culturales que, hacían imposible la existencia de una convivencia intercultural.

El alumnado quiso realizar una intervención con gran impacto a favor de la interculturalidad (VecinaMerchante, 2013). El alumnado compartió en este momento su intención de llevar a cabo actividades que fomentaran la concienciación social, para que no solamente las personas de este barrio reconocieran y aceptasen la diversidad existente y así, de manera preventiva, impedir la aparición de actitudes y expresiones discriminatorias de índole racista.

Igualmente, el alumnado contactó con diferentes agentes sociales de esta comunidad educativa (los servicios sociales y la comunidad de vecinos) para determinar la fecha de realización de la actividad, un mural colectivo. La voluntad del alumnado era difundir la realización de esta actividad mediante diferentes medios de comunicación y redes sociales con el único fin de provocar un impacto social.

Tabla 2. Resultados fase III

\begin{tabular}{|l|l|l|}
\hline Concepto & Actores & \multicolumn{1}{|c|}{ Idea Clave } \\
\hline & & $\begin{array}{l}\text { Desarrollar una } \\
\text { intervención para } \\
\text { erradicar los } \\
\text { perjuicios y los } \\
\text { estereotipos } \\
\text { relacionados con la } \\
\text { migración y crear un } \\
\text { barrio intercultural y } \\
\text { no simplemente } \\
\text { multicultural }\end{array}$ \\
& \multirow{5}{*}{ Intervención } & Alumnado \\
& & $\begin{array}{l}\text { Destruir el racismo } \\
\text { fundamentado en el } \\
\text { miedo a lo } \\
\text { desconocido }\end{array}$ \\
& & $\begin{array}{l}\text { Implicar al mayor } \\
\text { número de personas }\end{array}$ \\
& & $\begin{array}{l}\text { Generar impacto } \\
\text { mediático para } \\
\text { visibilizar la } \\
\text { experiencia realizada }\end{array}$ \\
\hline
\end{tabular}

Fuente: elaboración propia

Santamaría-Goicuria, I.; Corres-Medrano, I. y Arregi-Orue, J. (2021). El aprendizaje-servicio: una herramienta en la formación del profesorado en clave intercultural. Construyendo puentes entre Ecuador y Euskadi. RIDAS, Revista Iberoamericana de Aprendizaje Servicio, 11, 139-152.

DOI10.1344/RIDAS2021.11.10 


\subsection{Fase IV: evaluación del proceso}

Una vez finalizado el diseño y la implementación del proyecto de aprendizaje-servicio, la siguiente y última fase fue la de valoración y presentación de este. Este periodo de valoración se llevó a cabo en dos fases: la evaluación del 'aprendizaje' y la referente a la evaluación del 'servicio' realizado.

Por un lado, la evaluación del 'aprendizaje' de la experiencia, cuyo propósito era testar la adquisición de diferentes competencias por parte del alumnado, se realizó mediante un proceso dialógico en la universidad realizando una exposición del aprendizaje-servicio aplicado frente a un tribunal docente. El alumnado llevó a cabo un debate crítico sobre temas relacionados con el poder, los privilegios y las desigualdades sociales, examinando de forma crítica cuestiones como el racismo y la igualdad de oportunidades.

Por otro lado, la evaluación del apartado 'servicio', se realizó en la universidad con la asistencia de algunas personas participantes de la actividad, con el objetivo de recoger su grado de satisfacción en referencia a los objetivos fijados previamente. Tras el visionado del video realizado por los estudiantes sobre la creación del mural el intercambio de percepciones y opiniones realizadas a través de la sesión dialógica fue muy enriquecedora.

En la tabla 3 se recogen las valoraciones realizadas por todas las personas participantes en la actividad:
Tabla 3. Resultados fase IV

\begin{tabular}{|c|c|c|}
\hline Concepto & Actores & Idea clave \\
\hline \multirow{7}{*}{$\begin{array}{l}\text { Evaluación } \\
\text { proceso }\end{array}$} & \multirow{5}{*}{ Alumnado } & $\begin{array}{c}\text { El valor pedagógico } \\
\text { del aprendizaje- } \\
\text { servicio }\end{array}$ \\
\hline & & $\begin{array}{c}\text { Remarcan la } \\
\text { necesidad de conocer } \\
\text { a las culturas y la } \\
\text { importancia de la } \\
\text { creación de sistemas } \\
\text { de comunicación } \\
\text { (verbal, no verbal) } \\
\text { fundamentadas en el } \\
\text { entendimiento mutuo }\end{array}$ \\
\hline & & $\begin{array}{c}\text { La importancia de una } \\
\text { buena predisposición } \\
\text { para compartir y abrir } \\
\text { nuevos horizontes de } \\
\text { manera respetuosa y } \\
\text { crítica }\end{array}$ \\
\hline & & $\begin{array}{c}\text { El respeto hacia la } \\
\text { otra persona }\end{array}$ \\
\hline & & $\begin{array}{c}\text { Las ganas de } \\
\text { aprender desde y } \\
\text { para lo desconocido }\end{array}$ \\
\hline & $\begin{array}{l}\text { Participante } \\
\text { en la } \\
\text { experiencia }\end{array}$ & $\begin{array}{l}\text { Todo el mundo tiene } \\
\text { algo que aportar } \\
\text { independientemente } \\
\text { de la edad que se } \\
\text { tenga }\end{array}$ \\
\hline & $\begin{array}{l}\text { Participante } \\
\text { en la } \\
\text { experiencia II }\end{array}$ & $\begin{array}{c}\text { Este tipo de } \\
\text { actividades ayuda a } \\
\text { promover la } \\
\text { interculturalidad de la } \\
\text { zona }\end{array}$ \\
\hline
\end{tabular}

Fuente: elaboración propia

\section{Conclusiones}

La universidad comprometida socialmente debe apostar por una 
formación activa y de carácter interdisciplinar para el futuro profesorado, capaz de generar una sociedad para un mundo globalizado. Estas futuras generaciones responderán a los retos y desafíos que la sociedad contemporánea presenta encarnando un profesorado más activo y comprometido. Iniciativas como la descrita en esta investigación, comprometidas con la diversidad cultural global y también existente en el espacio social y cultural de nuestras ciudades, son capaces de iniciar procesos que faciliten la transformación social a través de la participación y formación del profesorado.

La puesta por el aprendizaje-servicio profundiza un cambio de paradigma en el que el alumnado es el principal actor de sus procesos de enseñanzaaprendizaje, abriendo nuevas posibilidades que cuestionan las posiciones hegemónicas y de relaciones de poder tradicionales fundamentadas en la adquisición y gestión del conocimiento. Esta metodología contribuye al desarrollo de la misión social de la universidad transfiriendo conocimiento útil para la transformación social, a través del desarrollo de proyectos docentes que generan una implicación y compromiso social, no solo a nivel individual sino también a nivel institucional. Por ello, se considera preciso contemplar la fortaleza de la metodología del aprendizaje-servicio como herramienta que responde a las necesidades de la sociedad contemporánea y se propone así utilizar este tipo de metodologías en el ámbito universitario, que discute las expectativas iniciales del pensar y del hacer.

Al comienzo de esta actividad formativa el alumnado mostraba un nivel de empatía, contacto real y conocimiento de la realidad multicultural de VitoriaGasteiz muy limitado. Este punto de partida reflejaba un escaso desarrollo de la competencia intercultural que se reducía a un vínculo teórico y lejano, carente de un marco experiencial, por tanto, incapaz de potenciar el resto de las herramientas conceptuales y prácticas necesarias (Deardorff, 2006; McKinnon, 2012).

Este trabajo, basado en el aprendizajeservicio, muestra cómo el alumnado supera esta situación inicial, desarrollando su competencia intercultural. En primer lugar, mediante el desarrollo de un seminario que fomentó el contacto intenso y directo con docentes indígenas ecuatorianos con los que debatieron sobre las claves en las que debe fundamentarse la educación intercultural en Ecuador y Euskadi. En segundo lugar, mediante el posterior desarrollo de una iniciativa del aprendizaje-servicio comunitaria, llevando a cabo una iniciativa de aprendizaje activo, de forma participativa y con relevancia social estableciendo contactos con asociaciones de migrantes, asociaciones de vecinos, centros educativos, asociaciones de Madres y Padres de Alumnos (AMPA), técnicos municipales. Fruto de esta experiencia el alumnado consiguió adquirir una competencia intercultural previamente inexistente.

Los procesos de enseñanza-aprendizaje fundamentados en el aprendizaje activo repercuten sustancialmente en la y Euskadi. RIDAS, Revista Iberoamericana de Aprendizaje Servicio, 11, 139-152. 
implicación y compromiso del futuro profesorado con la comunidad y por tanto son social y significativamente más relevantes formativamente. El poder que las metodologías como el aprendizaje-servicio poseen a nivel pedagógico, social, democrático y formativo del profesorado es relevante, especialmente, en contextos multiculturales, tanto a nivel de aula como de barrio-ciudad, porque 1) inciden: en aspectos personales y profesionales del alumnado 2) favorece el desarrollo de la competencia intercultural y el compromiso comunitario y una ciudadanía inclusiva y activa, que resultan fundamentales en su formación como futuros docentes y agentes facilitadores de transformación social. Además, resultan especialmente eficientes a la hora de desarrollar la mencionada capacidad intercultural, complementando el trabajo con fuentes secundarias, realizado en las aulas durante el desarrollo de la mención en Educación Intercultural, con el uso de fuentes primarias y testimonios directos.

El caso descrito también ofrece un valor añadido por haber incidido en los medios de comunicación vascos. Su presencia en los medios visibilizó el proyecto, concediendo una especial relevancia a nivel social al alumnado de la Facultad, a la comunidad del barrio participante, al tejido institucional y a los colectivos sociales de la ciudad.

Finalmente, destaca también su capacidad para integrar en la evaluación, junto al profesorado universitario, a los colectivos de migrantes participantes en la intervención educativo-social, que recibieron el testimonio directo de la experiencia de aprendizaje-servicio desarrollada. Este nivel de compromiso universitario y social no es habitual, y ofrece testimonio de la apuesta genuina a favor de la constitución de un espacio de diálogo entre saberes (Arregi et al., 2017) que acerca la universidad a la compleja sociedad intercultural de nuestros días.

\section{Referencias bibliográficas}

Alonso-Saéz, I., Arandia, M., Martínez, I., Martínez, B., y Gezuraga, M. (2015). El aprendizaje-Servicio en la innovación universitaria. Una experiencia realizada en la formación de educadoras y educadores sociales. Revista Internacional de Educación para la Justicia Social, 2(2), 195-216. Recuperado de https://revistas.uam.es/riejs/article/vie w/379/369

Aramburuzabala, P., y García, R. (2012). El aprendizaje-servicio en la formación de maestros. Recuperado de https://www.researchgate.net/publicati on/234163775 El aprendizajeServicio en la formacion de maestros

Arregi, J. (2011). Cuarto mundo: la acción exterior de los pueblos indígenas como instrumento de cambio $y$ reconocimiento internacional 1992 (Tesis doctoral). Universidad del País Vasco, Leioa, España.

Arregi, J., Saenz del Castillo, A. y Ugalde, A. (2017). Diálogos cruzados entre ciencia y conocimiento indígena. Desafíos para una investigación intercultural desde la didáctica de las ciencias sociales. En R. Martínez, R. y Euskadi. RIDAS, Revista Iberoamericana de Aprendizaje Servicio, 11, 139-152. 
García-Morís y C. García (Coords.) Investigación en didáctica de las ciencias sociales. Retos preguntas y líneas de investigación (pp. 313-323). Universidad de Córdoba y Asociación Universitaria del Profesorado de Didáctica de las Ciencias Sociales (AUPDSC). Recuperado de https://dialnet.unirioja.es/descarga/libr o/699144.pdf

Arregi, J., Saenz del Castillo, A. y Ugalde, A. (2018). De indígenas premodernos a guardianes de la tierra: el Sumak Kawsay como propuesta alternativa al sistema dominante. En B. Tosar, J. Pagès y A. Santisteban (Eds.) Que esta passant al món? Que estem ensenyant? Per un ensenyament de les ciències socials centrat en els problemes, la justícia social i la ciutadania global (pp. 197-204). Barcelona, España: Universitat Autònoma de Barcelona.

Arregi, J. y Orbea, G. (2016) Diálogos entre Ecuador y Euskadi en el marco de la Convención 169 de la OIT. En Z. Gaintza y J.J. Celorio (Coords.) Cooperando desde la UPV-EHU. Fortalecimiento del sistema de Educación intercultural bilingüe (pp. 183-195). Bilbao, España: Servicio Editorial de la Universidad del País Vasco.

Calatayud, M. A. (2006). Formación en educación intercultural: la voz del profesorado. Aula abierta, (88), 7384. Recuperado de https://dialnet.unirioja.es/descarga/arti culo/2684213.pdf

Chomsky, N., Barroso, J. M., y Flores, E. (2015). El capitalismo neoliberal puede ser combatido. Entrevista con Noam Chomsky. Iberoamérica Social: Revistared de estudios sociales, 3(4), 19-20.

Recuperado de https://iberoamericasocial.com/elcapitalismo-neoliberal-puede-sercombatido-entrevista-con-noamchomsky/

De Ferari, J.M. (2006). Responsabilidad social universitaria aproximación al concepto y a su práctica. En C. Fernández, C. Delpino y J. M. De Ferari, (Eds.), Responsabilidad social universitaria. Una manera de ser Universidad. Teoría y práctica de la experiencia chilena (pp. 41-43). Santiago de Chile, Chile: Proyecto Universidad: Construye País.

Recuperado de https://core.ac.uk/download/pdf/46752 393.pdf

Deardorff, D. (2006). Identification and assessment of intercultural competence as a student outcome of internationalization. Journal of studies in international education, 10(3), 241266. doi: $10.1177 / 1028315306287002$

Delors, J. (1996). La Educación encierra un Tesoro: Informe a la UNESCO de la Comisión Internacional sobre la Educación para el Siglo XXI.

Compendio. Madrid, España: Santillana.

Essomba, M. (2015). Una formación crítica de los profesionales de la educación al servicio de la diversidad cultural y la interculturalidad. En A. Escarbajal (Ed.), Comunidades interculturales y democráticas (pp.127142). Madrid, España: Narcea

Francisco, A. y Moliner, L. (2010). El

Santamaría-Goicuria, I.; Corres-Medrano, I. y Arregi-Orue, J. (2021). El aprendizaje-servicio: una herramienta en la formación del profesorado en clave intercultural. Construyendo puentes entre Ecuador y Euskadi. RIDAS, Revista Iberoamericana de Aprendizaje Servicio, 11, 139-152.

DOI10.1344/RIDAS2021.11.10 
Aprendizaje Servicio en la Universidad: una estrategia en la formación de ciudadanía crítica. Revista electrónica interuniversitaria de formación del profesorado, 13(4), 69-77. Recuperado de

https://www.redalyc.org/pdf/2170/217 015570006.pdf

Giroux, H. (2019). Hacia una pedagogía de la esperanza educada bajo el capitalismo de casino. Pedagogía y Saberes, (50), 153-158.

doi: $10.17227 /$ pys.num50-9508

Lucas, S. y Martínez-Odría, A. (2012). La implantación y difusión del Aprendizaje-Servicio en el contexto educativo español. Retos de futuro de una metodología de enseñanzaaprendizaje para promover la innovación en la Educación Superior. Revista del Congrés Internacional de Docència Universitària i Innovació, CIDUI, (1). Recuperado de https://www.cidui.org/revistacidui/inde x.php/cidui/article/view/122/110

Martínez, B., Martínez, I., Alonso, I. y Gezuraga, M. (2013). El aprendizajeservicio, una oportunidad para avanzar en la innovación educativa dentro de la Universidad del País Vasco. Tendencias pedagógicas, 21, 99-118. Recuperado de

https://revistas.uam.es/tendenciaspeda gogicas/article/view/2027/2134

Martínez, M. (2008). Aprendizaje servicio y responsabilidad social de las universidades. Barcelona, España: Octaedro.

McKinnon, H. (2012). What is intercultural competence? Recuperado de

https://www.qcu.ac.uk/media/qcalwebv 2/theuniversity/centresprojects/globalp erspectives/Definition of Intercultural competence.pdf

Palomero, P. (2006). Inmigración y educación en la ciudad de Zaragoza: análisis de la realidad y propuestas para una escuela intercultural. Revista interuniversitaria de formación del profesorado, (55), 91-112. Recuperado de

https://dialnet.unirioja.es/descarga/arti culo/2234430.pdf

Polkinghorne, D. E. (2005). Language and meaning: Data collection in qualitative research. Journal of Counseling Psychology, 52(2), 137-145. doi:10.1037/0022-0167.52.2.137

Puig, J. M. (2009). Aprendizaje servicio (ApS). Educación y compromiso cívico. Barcelona, España: Graó.

Puig, J. M., Batlle, R., Bosch, C. y Palos, J. (2007). Aprendizaje servicio. Educar para la ciudadanía. Barcelona, España: Octaedro.

Puig, J. M., Martín, X. y Batlle, R. (2008). Com començar una experiència d'aprenentatge servei. Barcelona, España: Centre promotor d'APS en Catalunya.

RMC Research Corporation. (2006). K12 Service-Learning Project Planning Toolkit. Scotts Valley, CA: National Service-Learning Clearinghouse.

Rubio, L. y Escofet, A. (Coords.) (2017). Aprendizaje-servicio (ApS): claves para su desarrollo en la y Euskadi. RIDAS, Revista Iberoamericana de Aprendizaje Servicio, 11, 139-152. 
Universidad. Barcelona, España:

Octaedro.

Sotelino, A., Santos Rego, M. y Lorenzo, M. (2016). Aprender y Servir en la

Universidad: Una vía cívica al desarrollo educativo. Teoría de la Educación, 28(2), 225-248. doi:

$10.14201 /$ teoredu282225248

Vecina-Merchante, C. (2013). La acción comunitaria, un camino para la integración social y la interculturalidad. Trabajo Social GlobalGlobal Social Work, 3(5), 54-75.

doi: $10.30827 /$ tsg-gsw.v3i5.1528 y Euskadi. RIDAS, Revista Iberoamericana de Aprendizaje Servicio, 11, 139-152. 